\title{
Review
}

\section{Individual Differences in Language Acquisition and Processing}

\author{
Evan Kidd, 1,2,3 Seamus Donnelly, 2,3 and Morten H. Christiansen ${ }^{3,4,5,6, *}$
}

Humans differ in innumerable ways, with considerable variation observable at every level of description, from the molecular to the social. Traditionally, linguistic and psycholinguistic theory has downplayed the possibility of meaningful differences in language across individuals. However, it is becoming increasingly evident that there is significant variation among speakers at any age as well as across the lifespan. Here, we review recent research in psycholinguistics, and argue that a focus on individual differences (IDs) provides a crucial source of evidence that bears strongly upon core issues in theories of the acquisition and processing of language; specifically, the role of experience in language acquisition, processing, and attainment, and the architecture of the language system.

\section{Individual Differences: An Inconvenient Truth}

Humans differ in every way imaginable, but in the psychological sciences the treatment of IDs has historically been uneven. On the one hand, correlational research, as exemplified by the study of intelligence and personality, assumes the existence of meaningful IDs that can be measured and quantified using psychometric methods. On the other hand, the more-familiar experimental approaches of the cognitive sciences have tended to ignore individual variability in favor of the study of group-based differences. This is despite clear IDs in basic processes underlying much of cognition, such as attention, memory, and language [1-3].

Despite their ubiquity, IDs represent something of an inconvenient truth: their presence is undeniable but our theories and experimental methods overwhelmingly downplay their importance (e.g., by relegating them to error variance). This approach results in theories that postulate idealized and mostly invariant human cognitive capacities [4], which has no doubt ensured steady scientific progress but leads to the mistaken assumption that cognitive capacities are subject to less variation than is in fact the case. Dissatisfaction with this state of affairs is not new. Estes [5] warned against the use of group data to extrapolate to individual functions, and there have been repeated calls to integrate correlational and experimental methods (e.g., [6-8]). Here, we focus on IDs in language, a field in which there has been renewed interest in individual variation. We argue that a focus on IDs in language ability has the potential to shed new light on longstanding theoretical debates, and bring us closer to a detailed mechanistic understanding of human language.

\section{Theoretical Perspectives on Individual Differences}

The study of IDs has the potential to clarify theoretical controversies within the language sciences. If IDs exist, our theories of language acquisition (see Glossary) and language processing must account for them. More specifically, we argue that our theories must be able to account for three different types of empirical observation. First, and most obviously, our
Highlights

IDs are a pervasive feature of language acquisition and processing. Whereas IDs have been the focus of studies of, for instance, neurocognitive disorders, there is now an increasing focus on IDs across the entire spectrum of abilities.

IDs are evident across all components of the linguistic system, from the acquisition and processing of properties of speech, to complex grammatical structures and discourse.

IDs result from a complex interplay of endogenous cognitive systems and the environment. Understanding the contribution of these variables allows a better understanding of the mechanisms underlying human language.

Different theoretical approaches to language acquisition and processing make differing predictions regarding the nature of IDs in the population; therefore, IDs studies can contribute significantly to longstanding debates in the language sciences.

${ }^{1}$ Language Development Department, Max Planck Institute for Psycholinguistics, Nijmegen, The Netherlands

${ }^{2}$ Research School of Psychology, The Australian National University, Canberra, Australia

${ }^{3} A R C$ Centre of Excellence for the Dynamics of Language, Canberra, Australia

${ }^{4}$ Department of Psychology, Cornell University, Ithaca, NY, USA

${ }^{5}$ School of Communication and Culture, Aarhus University, Arahus, Denmark

${ }^{6}$ Haskins Laboratories, New Haven, CT, USA 
theories must allow for the existence of IDs, where observed (the 'existence' imperative). Second, our theories must account for observed relationships between language and the amount and nature of input (the 'environmental' imperative). Third, and perhaps most informatively, our theories must account for observed relationships between linguistic subsystems and across cognition more generally (the 'architectural' imperative).

This latter point deserves some elaboration. In their early work on IDs in language acquisition, Bates and colleagues [9] drew on the hypothesized distinction between vertical and horizontal faculties to theoretically frame the study of IDs within the correlational method. Vertical faculties are modular, domain-specific processes that are specialized for one kind of content (e. g., language or face processing), whereas horizontal faculties are domain-general cognitive systems that operate on multiple types of content (e.g., memory or executive function) [10]. Given the different assumptions regarding how the content of vertical and horizontal faculties interact across the entire cognitive architecture, patterns of association and dissociation can provide evidence for common or distinct underlying mechanisms, or in the words of Bates and colleagues, 'the seams and joints' of the language system ([9] p. 11). If components of language hang together (e.g., vocabulary and grammar), there is evidence for a common underlying mechanism, and if they do not, then multiple distinct mechanisms may be at play. A similar logic holds for the relationship between language and other cognitive systems. Thus, a focus on IDs provides a unique perspective on longstanding debates in psycholinguistics, in particular, the degree of interaction between hypothesized components of the linguistic system and the relationship between language and other cognitive systems.

Here, we consider how current IDs research bears upon the three ID imperatives and, thus, how an ID approach can constrain theories of language acquisition and processing. First, we discuss two broad theoretical perspectives on language acquisition and processing, highlighting the predictions they make about the existence and patterns of IDs. We then review empirical research on language acquisition and processing, highlighting: (i) the existence of IDs; (ii) their relationship to the input; and (iii) their relations to other linguistic and cognitive factors. We then discuss the implications of these IDs for theories of processing and acquisition.

\section{Theoretical Traditions in Language Acquisition and Processing}

Different theoretical traditions within language acquisition and processing make different predictions about each of the three questions described above. Traditional formal linguistic approaches, which have typically assumed vertical faculties, predict IDs for vocabulary but not readily for formal components of the language system. In this approach, IDs in other linguistic domains, such as grammar, are largely attributed to performance limitations caused by variation in external cognitive systems that interface with, but are separate from, language [e.g., working memory (WM)]. The argument is that the implementation of formal rules is largely invariable and not particularly influenced by IDs elsewhere in the system (e.g., vocabulary), but can be perturbed in instances of high computational burden. There is some debate regarding how systematic an effect IDs in external cognitive systems may have on language processing (e.g. , [11,12]). Suffice to say, insofar as language must be encoded and processed in real time, variations in sensory and cognitive processing have some role [13]. However, the crucial point is that such effects do not shape the core representational properties of the linguistic system.

In acquisition, the formal approach assumes the existence of abstract innate knowledge of language at birth (i.e., linguistic principles), coupled with formal computational machinery that enables structure building. This constitutes universal grammar (UG), which historically has only been predicted to be subject to IDs in exceptional cases (e.g., neurocognitive disorders
${ }^{*}$ Correspondence: christiansen@cornell.edu (M.H. Christiansen). 
[14]). However, recent proposals incorporate concepts that may predict IDs. Experience with language is argued to affect language and, by implication, variation in input may in principle result in variable rates of development [15]. Thus, differences in rates of occurrence of individual syntactic phenomena in the input (e.g., wh-questions in English) could result in variations in age of acquisition because they will increase or decrease the rate in which the language-specific grammatical options from UG are identified, or even lead children to set different grammatical options. Other 'third factors', which are once again external to UG but are implicated in the process of acquiring a native language, are also argued to have a role. Several proposals exist, such as statistical learning (SL), inductive inference, and 'computational efficiency' $[15,16]$. However, in many cases, the influence of these third variables is expected to be minor, especially where UG constrains the child's hypothesis space and, therefore, differences in final attainment are not expected.

Overall, formal approaches predict a variable profile of IDs in the linguistic system. IDs in vocabulary are expected because words are specific to individual languages and, therefore, must be learnt. However, IDs in formal systems, such as grammar, are expected to be comparatively minimal because, as vertical faculties, the range of variation across development is restricted by innate knowledge structures and the largely invariant nature of the end state.

At the other end of the theoretical spectrum, emergentist approaches to language, such as the usage-based approach to acquisition [17] and experience, or constraint-based approaches to language processing $[18,19]$, differ from formal approaches on several dimensions and, therefore, predict a different pattern of individual differences. Most clearly, these approaches place a larger emphasis on the input in both acquisition and adult processing, and claim that language must be largely learnt via analyses of, and generalization from, the input. In acquisition, this means that children must induce knowledge about key properties of the language with little language-specific prior knowledge. The approach does not assume sharp boundaries between linguistic subsystems (e.g., [18-22]); for instance, unlike formal theories, emergentist theories assume a tight integration of form and meaning (i.e., syntax and semantics). They also predict meaningful interactions between levels of language that are themselves subject to IDs. One commonly reported interaction is between vocabulary and grammar [23,24]. In acquisition, vocabulary development is closely coupled with early grammatical development. The emergentist explanation is that grammatical generalisations are made over vocabulary items and, thus, IDs in vocabulary development will affect the rate of grammatical development. For other structures, the frequency of vocabulary items guides both acquisition and grammatical processing (e.g., $[25,26])$, the implication being that the acquisition and implementation of grammatical routines could be affected by IDs in experience with individual vocabulary items across different syntactic environments.

The emphasis that emergentist approaches place on the input necessitates the existence of learning mechanisms powerful enough to make the right kind of generalizations from the input $[20,27]$. Such mechanisms may also be subject to individual variation, which, in combination with differences in the input, will jointly determine IDs in language acquisition and, ultimately, adult processing and attainment. Crucially, although any account of language must specify what mechanisms enable humans to learn from their input, exactly what learning mechanisms support the analysis of the input and how they do so is currently unclear. Therefore, mapping variation in both candidate mechanisms and the target system is crucial to theory building and testing in the language sciences.

\section{Glossary}

Executive function (EF): domaingeneral control functions that regulate thought and action.

Emergentist approaches: a broad class of approaches to language that eschew claims of innately specified knowledge (see 'universal grammar') and instead argue that language is learnable via multiple mechanisms that may or may not be specific to language.

Formal linguistic approaches: a class of approaches to language that assume linguistic structure is independent of meaning and usage. Horizontal faculty: a multipurpose cognitive system that processes information across several domains, such as memory or executive function (contrasted with vertical faculty)

Language acquisition: the process by which speakers acquire the ability to process a target language. Here, the term is used to refer to first (i.e. native) language acquisition.

Language processing: the rapid use of linguistic representations to comprehend and produce language. Morphosyntactic paradigm: the set of related forms that a basic lexcial unit (such as a word) can take when inflected for different grammatical features.

Statistical learning (SL): the process of tracking and learning $\mathrm{co}$ occurrences between elements in the environment. These distributional relationships can involve both adjacent and nonadjacent elements. Universal grammar (UG): a hypothesized innate endownment of language-specific constraints that support the acquisition of all existing languages.

Vertical faculty: a neural module specialized for processing information for a particular domain only, such as faces or language (contrasted with horizontal faculty). Working memory (WM): the storage and manipulation of mental representations in conscious awareness. 


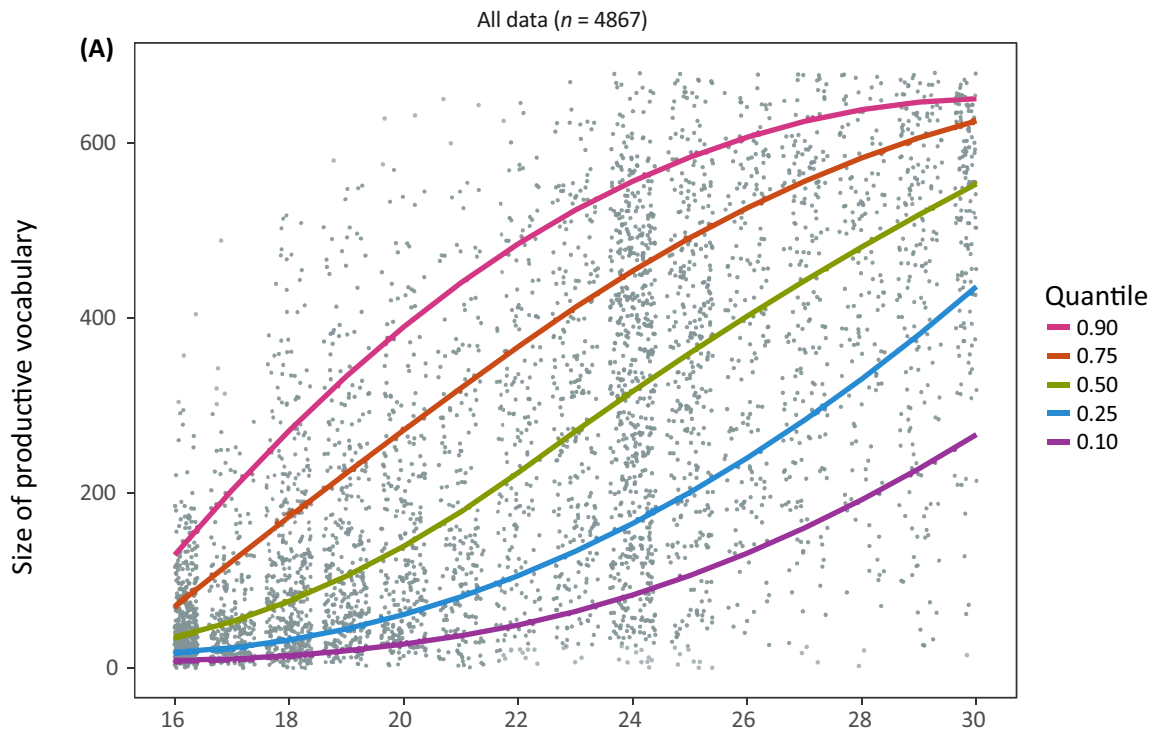

Age (months)

(B)

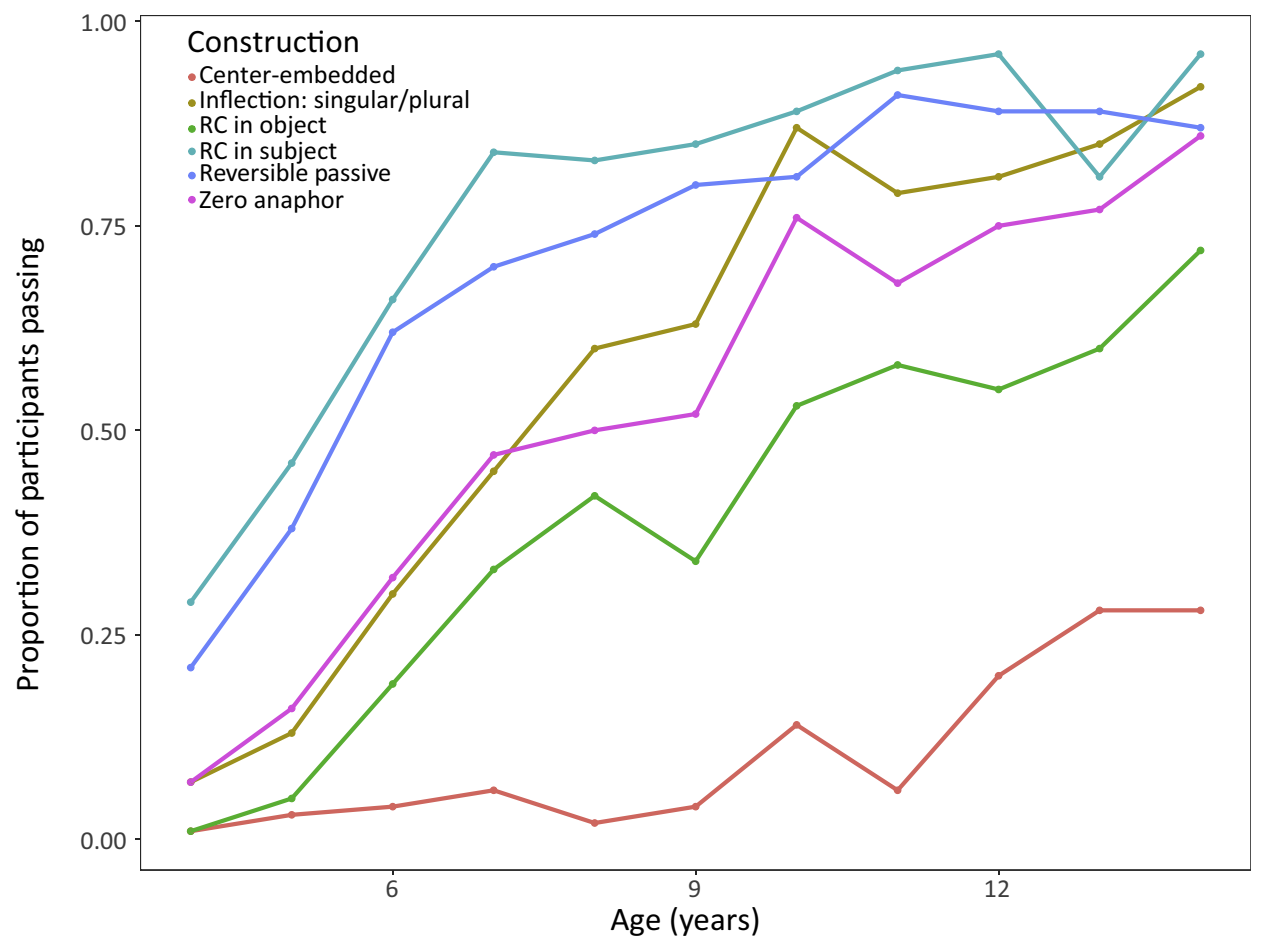

Trends in Cognitive Sciences

Figure 1. Individual Differences (IDs) in Language Acquisition. (A) Cross-sectional MacArthur-Bates Communicative Development Inventory vocabulary production data from 4687 English-speaking children aged 16-30 months [37]. This is a checklist where caregivers mark whether their child produces certain words and communicative behaviors. As can be seen, there are substantial IDs both within age bands and across developmental time. (B) Norming data (4-15 years) for several English structures on a popular standardized test of grammatical knowledge: the Test for the Reception of Grammar (2nd edition) [130]. The y-axis indicates the proportion of children who performed at ceiling. As can be seen,

(Figure legend continued on the bottom of the next page.) 
Overall, emergentist approaches predict a more widespread pattern of IDs due to the greater emphasis placed on the input and learning mechanisms in jointly contributing to language acquisition and use. Strong patterns of interaction both within the linguistic system and between language and other cognitive systems are expected. Therefore, although we have inevitably used broad brushstrokes, it is clear that different approaches to language make different assumptions regarding our three ID imperatives and, thus, that they predict different patterns of IDs. We next review the current evidential base bearing upon these three imperatives.

\section{Individual Differences Are Pervasive in Language}

The study of IDs in language has traditionally been confined to a focus on either language proficiency in atypical circumstances (e.g., acquired and developmental language disorders, or second language learning), or aspects of language that all researchers agree are subject to significant learning and that are influenced by environmental variation (i.e., vocabulary) [28,29]. However, there is growing acknowledgement that IDs are evident across the entire population and across the entire linguistic system (Figures 1 and 2).

Indeed, IDs in language proficiency are the norm rather than the exception. In first language acquisition, IDs are large and notably stable across development [30,31]. They are also observed early and across all domains. For instance, variation in auditory brainstem responses in 6-week-old infants predicts their emerging language knowledge at 9 months [32]. Similarly, resting-state brain activity in parietal areas at birth correlates with language comprehension at 15 months [33]. These early differences may be related to subsequent variability in the development of native language knowledge. Both the ability to make phonetic distinctions and segment words from running speech develop within the 1st year of life, but are subject to significant interindividual variation. Notably, longitudinal studies show that children who master these skills early have better vocabulary knowledge months or even years later compared with children who master them at an older age [34-36].

The downstream effects of these early developments are clear in children's variable vocabulary and grammatical development. Initial vocabulary development is slow, but the rate at which children acquire vocabulary soon differentiates fast from slow (and average) developers. For instance, vocabulary production norms from Wordbank shows that a child in the 90th percentile at 16 months knows the same number of words as a child in 10th percentile at 26 months [37]'. Perhaps more surprisingly, children's grammatical competence also varies across development [38]. Although it has received scant attention, there also appear to be significant individual differences in children's pragmatic development [39].

IDs do not disappear after childhood. In adults, there are clear IDs in both online language processing and ultimate attainment. The quality of lexical representations predicts individual differences in several aspects of reading [40,41], and may lead to the development of qualitatively different reading strategies over an individual's development [42]. There are also significant individual differences in syntactic processing, including in the processing of relative

several structures are still not fully mastered at the group level even in adolescence. Center-embedded sentences are those in which a full clause separates the subject from the main verb (e.g., 'The cat the dog sees is running'). Inflected singular/plural sentences contain one singular and one plural noun (e.g., 'The cats are next to the dog'). Relative clauses $(\mathrm{RC})$ in Object sentences are those in which a RC follows the object (e.g., 'The dog chases that cat that is brown'). RC in Subject sentences are those in which a RC follows the subject (e.g., 'The dog that is brown is chasing the cat'). Zero anaphor sentences comprise two clauses with no surface-level subject in the second clause (e.g., 'The dog is looking at the cat and is jumping'). 
(A)

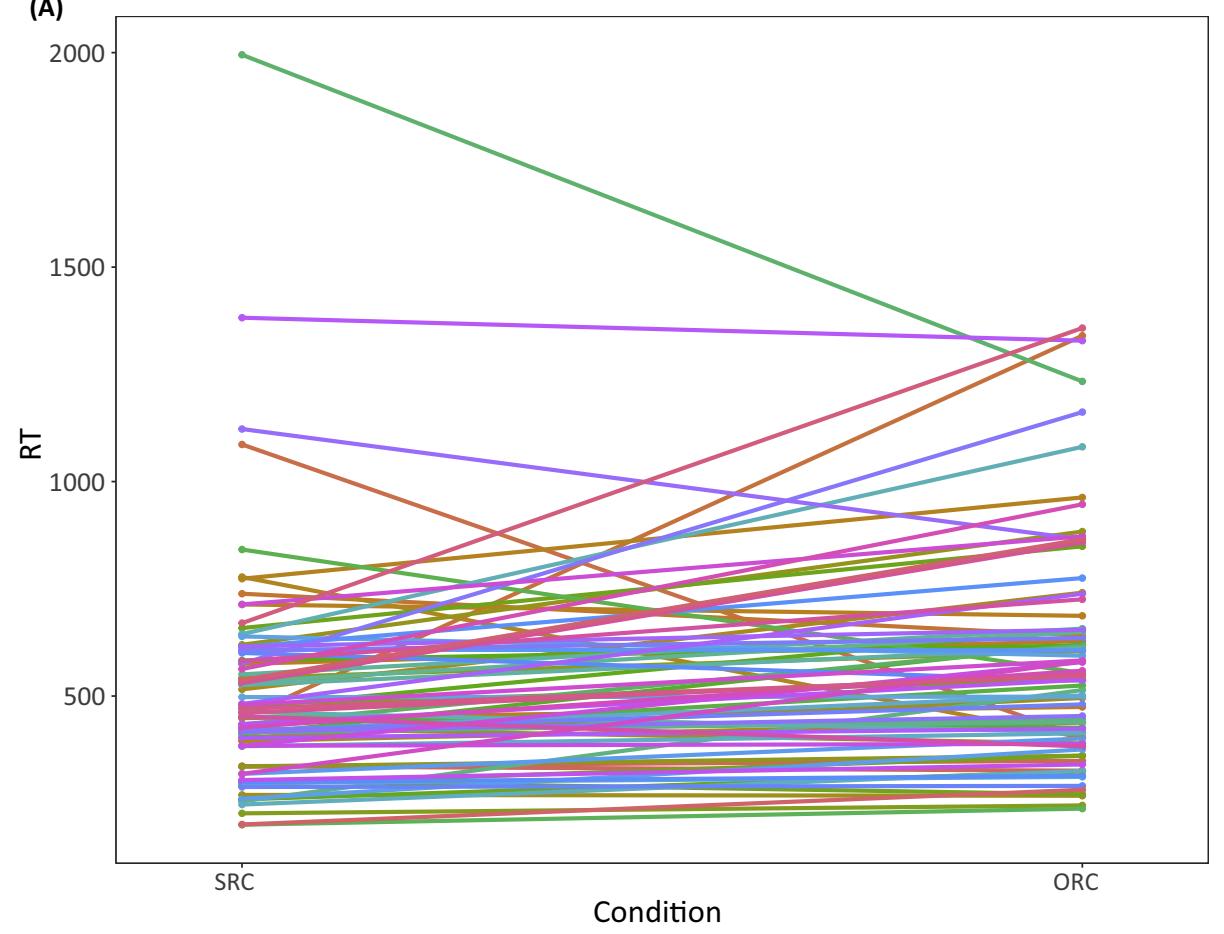

(B)

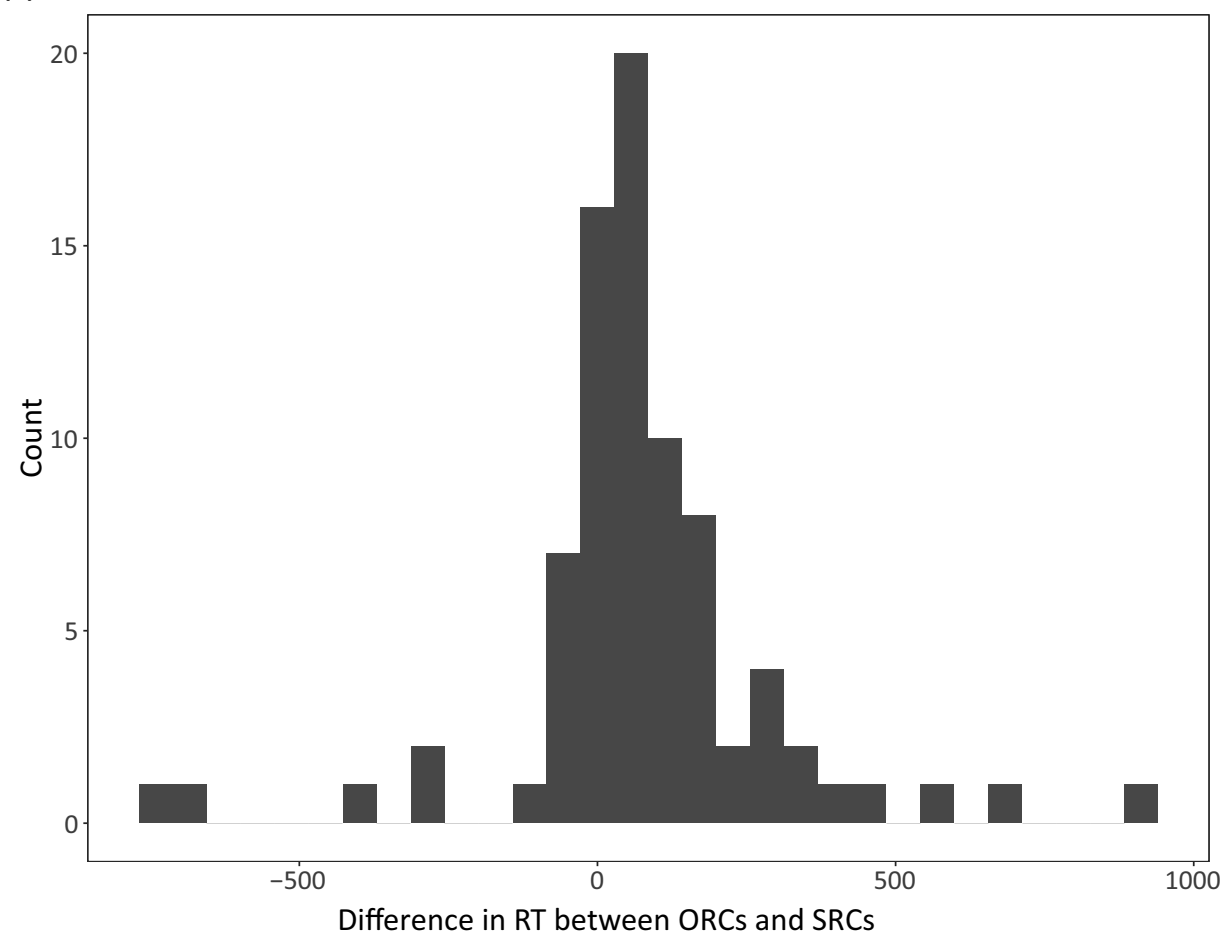

Trends in Cognitive Sciences

Figure 2. Individual Differences (IDs) in Online Sentence Processing. (A,B) Combined data from two self-paced reading experiments [111,112] $(n=80)$ that investigated adults' processing of center-embedded subject ('the boy that 
clauses [43], resolving syntactic ambiguities [1,44], using contextual information for ambiguity resolution [45], and interpreting pronouns [46]. Variability is reliably observed at the neurological as well as the behavioral level $[47,48]$. More recently, there have been demonstrations of IDs among typically developing adults in ultimate attainment [49]. This research shows that individuals can draw very different generalizations for complex morphosyntactic paradigms (e.g., the Polish genitive [50]), or consistently misinterpret some relatively low-frequency constructions, such as the English passive (e.g., 'the boy was chased by the girl' [51]).

\section{Relationships between Language and the Environment}

Every aspect of language representation and use is potentially affected by environmental variables pertaining to how language is used and more-distal variables that affect cognition or social interaction. The most-immediate environmental effect on language pertains to the frequency of language use, which affects both acquisition and adult language processing $[52,53]$. All things being equal, more-frequent words and structures are typically acquired earlier and are processed more easily (but there also appears to be a special facilitation for early acquired constructions [54]). Accordingly, variation in input quantity (i.e., the amount of language children hear) significantly influences acquisition [29]. However, variation in input quality also has a role. Several indicators of quality, including lexical diversity, use of decontextualized language, and properties of verbal and nonverbal interaction are also important [5557], with computational modeling showing that some quality indicators, such as lexical diversity, are more important than input quantity [58].

One commonly cited variable that affects input quantity and quality is socioeconomic status (SES). Specifically, work that has predominantly investigated North American families has shown that children from lower SES backgrounds tend to receive less input overall, less diversity in vocabulary, and are engaged less in extended conversation $[59,60]$. SES differences extend beyond spontaneous speech, with differences also identified in home language environments (e.g., book reading practices [61]). These differences have significant effects on both vocabulary and grammatical development [62,63] and are consistent with studies showing a relationship between the nature of caregiver-child interaction and subsequent language development [64]. Moreover, maternal SES even predicts subsequent adult language processing in college-aged participants [65]. SES also has an effect on language via nonverbal communication, with children's early use of gesture partially mediating the relationship between SES and vocabulary at school entry [66]. Interestingly, the negative consequences of low SES can be mitigated by good endogenous skills for language, such as SL [67].

SES is best construed as a macro proxy variable indexing variation in the environment. Thus, it is important to acknowledge that much of the work on SES and language acquisition has been conducted in North America, which limits the generalizability of the findings [68]. Crosscultural

pushed the girl . . . ') and object relative clauses ('the girl that the boy pushed . . . '). In the experiments, participants read a single word at a time, pressing a button to proceed to the next word. Typically, there is an increase in reaction times (RTs) at points of high syntactic complexity. Relative clauses (RCs) are one of the most-common structures used to study IDs in adults; at the group level, there is typically an advantage for processing subject RCs. (A) Each participants' median reaction time in the critical time window (main verb) for subject (SRC) and object (ORC) RCs. As can be seen, there is large variability between participants in median reading time for each clause type as well as large variability between participants in the difference in RTs between ORC and SRC. This is confirmed by (B), which plots the distribution of difference scores (RTs for ORC sentences - RTs for SRC sentences) for the entire sample. While participants on average read the critical word in SRC sentences $58 \mathrm{~ms}$ faster than the one in ORC sentences, across participants this difference ranged from $-500 \mathrm{~ms}$ to nearly $1000 \mathrm{~ms}$. 
work demonstrates significant variation in language socialization practices [69]. However, currently there is not enough data from other cultures to begin to grasp the effects of such variation, and the field will benefit significantly from more crosscultural comparisons [70]. Note also that so-called 'SES effects' are not solely reducible to cultural influences; they are also linked to a host of environmental factors (e.g., diet [71] and activity [72]) that affect brain development [73].

In adults, SES, as measured by education level, is associated with differences in attainment [49-51]. These differences occur largely in low-frequency structures that are more common in written language (e.g., the English passive; sentences containing quantifiers, e.g., "every hat has a rabbit in it') and, thus, may reflect differences in exposure to print [74]. This in turn suggests that variability in language proficiency is at least partially linked to expertise and the cognitive niches that individuals seek out. Accordingly, we see greater language proficiency in individuals who work in language-rich environments (e.g., language teachers and simultaneous interpreters [75]).

The small literature on environmental effects on adult language processing and attainment highlights the typically Western Educated Industrialized Rich Democratic (WEIRD [76]) nature of samples in adult psycholinguistic research. This suggests that the likely range of IDs is larger than what might be estimated from our current evidential base, which mostly draws on samples of university undergraduates. It will be important to continue to test more-representative samples if we are to chart and explain the full range of IDs in the general adult population.

\section{Relationships between Linguistic and Cognitive Variables}

One promise of IDs research is that the pattern of associations and dissociations between linguistic subsystems and between linguistic and cognitive tasks can reveal properties of the underlying architecture of the language system [9]. It has long been known that IDs across linguistic subsystems are reliably related to one another. For example, in language acquisition, vocabulary size is related to how fast children access words online [77], and is a strong predictor of grammatical development (e.g., $[30,78,79])$. In adult language processing, variability in vocabulary knowledge predicts spoken lexical access in challenging conditions (e.g. $[80,81])$, and lexical frequencies can influence syntactic processing [82].

Perhaps more revealing, a significant body of research also shows associations between language and other cognitive tasks. Human memory, for example, is a complex set of systems that, pretheoretically, are likely to support language acquisition and use. WM has long been implicated as a strong candidate because it systematically varies within the population and correlates with language. For instance, variation in phonological short-term memory correlates with children's vocabulary development and is an accurate marker of language impairment $[83,84]$. Similarly, both verbal WM and visuospatial WM have been linked to syntactic processing [e.g., [85,86]). WM tasks have been shown to have moderate genetic and nonshared environmental components $[87,88]$.

One issue concerns exactly how WM relates to language. One possibility is that WM capacity systematically varies within the population, which captures the intuition that human cognition is naturally capacity limited. However, more-recent work suggests that performance on verbal WM itself is dependent on long-term representations for language mediated by language experience (e.g., [43,89,90]). Therefore, a second possibility is that variation in WM measures, which often contain linguistic content, reflect differences in ease of retrieval (and, presumably, 
encoding), which may in part stem from variation in vocabulary knowledge [91]. This possibility is consistent with models that ground language processing within independently motivated principles of memory, where an extremely limited active memory span leads to difficulties in the retrieval of lexical items causing comprehension difficulties [13,92]. Explicit models of how different components of memory interact and how this affects language are a priority (e.g., $[19,93])$.

Another cognitive capacity consistently linked to performance on linguistic tasks is executive function $(\mathrm{EF})$ : a set of domain-general control functions for regulating thought and action. Examples include inhibition, switching, and updating, among others [94]. Performance on EF tasks varies substantially across the population and is heritable [95]. Many EF tasks involve competition between representations or responses. Since it is assumed that linguistic representations compete during both comprehension and production, it should not be surprising that IDs in EFs predict many aspects of language processing. For example, they predict performance on recovery from garden-path sentences in both adults [96,97] and children [98]; interference from locally coherent but globally inappropriate lexical items during sentence processing [99]; pragmatic comprehension and production [100]; lexical ambiguity resolution in children [101]; and interference across languages in bilingual speakers [102].

Interestingly, WM and EF are interrelated (see Outstanding Questions). For example, one prominent account of WM views this construct as the interaction between executive attention and long-term memory [103]. In this account, one source of IDs in WM capacity reflects individual differences in domain-general executive attention (in addition to variation in domainrelevant memory traces, e.g., long-term memory for words). This same executive attention factor may be involved in EF tasks. Consistent with this perspective, IDs data suggest that performance on WM tasks and a battery of EF tasks load onto a single executive attention factor. Therefore, while these two constructs are potentially separable, they may reflect IDs in a more-fundamental attentional process [104].

WM and EF describe largely explicit and sometimes effortful cognitive processes, but much knowledge of language is implicit and potentially probabilistic. For instance, languages have unique distributional properties to which speakers attend to both acquire and process language. Such cues may be local (e.g., that, in English, a determiner such as 'the' will often occur adjacent to a noun, as in 'the students'), but can also involve long sequences, where dependencies are established across large amounts of intervening material (e.g., 'the students who the professor liked to teach during the fall semester were always on time for class'). These probabilistic cues may be analyzed via SL, the process of using co-occurrence to group elements in the environment. IDs in SL have been linked to language proficiency across the lifespan, a relationship that has been attested across multiple domains of language, including vocabulary [105], grammatical [106], and literacy development in children [107]; and second language-learning adults [108]. The link is also attested in adults, and appears most strongly in tasks involving predictability; for instance, in predicting likely continuations of sentences in context [109] or in grammatical sequencing [110-112]. Similar effects have been observed in adult readers' use of orthographic cues for lexical access during reading [132]. Box 1 provides a more in-depth overview of the relationship between SL and language. 
Box 1. IDs in Statistical Learning and Their Relationship to Language

SL underlies our ability to acquire the underlying distributional regularities present in an often messy environment. Since the first demonstration that young infants were capable of discriminating between trained and untrained words in an artificial miniature language after only a 2-min exposure [131], SL has been put forward as a potential alternative to formal approaches to acquisition. Specifically, SL is suggested to provide a potentially powerful mechanism with which children can acquire language from input without strong assumptions about innateness (although see [16]). More recently, the field has attempted to quantify IDs in SL, and has linked these to language proficiency in children and adults [105-112,132]. This work has shown some promise, with mostly medium-positive associations between SL and language typically reported [133]. However, there are several outstanding theoretical and methodological questions. Methodologically, it is unclear exactly what SL tasks measure, and whether they do so reliably. Most studies to date have used SL tasks that were designed for group-level comparisons, and do not meet standards required for reliable psychometric tests [134]. Additional methodological problems concern what statistical properties are measured and the modality of presentation. Typically, studies quantify SL as the ability to learn simple transitional probabilities, but SL-forlanguage likely requires more than this, and even appears to be significantly influenced by the target language [135], thus suggesting a dynamic SL mechanism that is subsequently shaped by environmental input. The theoretical issues intersect with the methodological ones. Demonstrations that SL in the visual domain correlates with language led to suggestions for an underlying domain-general capacity supporting language [109]. However, this claim is weakened by lack of correlations between SL conducted in the visual and auditory domain [134]. Thus, it appears that SL is not a unitary mechanism, but may be a set of modality-constrained computational principles [136]. These computational principles may be shared across modalities (thus accounting for cross-domain effects in IDs studies), but SL will inevitably be constrained by modality-specific and neurologically distinct encoding procedures and representations. Overall, the research to date suggests that SL tasks are capturing cognitive skills important for language, but it is still unclear exactly what they capture [133]. Fortunately, new methods are emerging that may support further advancement in this area $[137,138]$. Therefore, future research will be better able to estimate the relative contribution that SL makes to language.

\section{Theory Testing and Development within ID Designs}

The evidence reviewed in the previous section constitutes a range of empirical facts for which all theories of language acquisition and processing must account. What is clear from the past IDs research is that: (i) IDs are pervasive across the language system throughout the lifespan; (ii) IDs are related to environmental variables, such as the quantity and quality of the input; and (iii) IDs in linguistic tasks are related to other linguistic subdomains and other cognitive functions, such as WM, EF, and SL. Given that language is a complex dynamic system [113], it is difficult to see how it could be any other way. Overall, the current state of the literature points to a substantial degree of interactivity both within language subsystems as well as between language and other cognitive systems. Therefore, the data do not support the most-extreme modular approaches to language. However, beyond not supporting a radical non-interactionist account, the current evidence is consistent with a range of possible architectural solutions. A greater focus on IDs will be crucial for testing current accounts and for developing future theoretical models.

Correlational research is not the only way IDs can be used for theory testing; examining the structure of IDs on individual tasks may also help clarify theoretical debates in language acquisition and processing. For example, both formal and emergentist accounts of language acquisition predict that, from a relatively young age (around 2 years), English-speaking children have acquired knowledge of word order to interpret basic transitive sentences (e.g., 'The bunny gorped the frog'), and should perform above chance at the group level on experimental tests of the structure (e.g., [114,115]). However, the two accounts make different predictions about the structure of individual differences. The formal account assumes that children have latent abstract syntactic representations that, once triggered by the input, are immediately productive [116]. Accordingly, the distribution of individual differences in experimental tests of this structure should be bimodal, with some children performing above chance, and some performing near chance, and any other difference between children being due to measurement error. 
However, emergentist accounts (e.g., usage-based approach) assume that children are gradually constructing abstract representations of syntax from their input, and, therefore, there should be graded individual differences between participants [117]. That is, the distribution of individual differences should be unimodal and more variable than that predicted by the formal account.

A similar logic holds for studies with adults. There has been considerable recent debate regarding whether grammatical judgments are categorical or probabilistic [118-122]. As in the acquisition example above, the two explanations predict different patterns of IDs. Given the right methodology, the formal approach predicts categorical judgments and, therefore, a bimodal distribution of grammatical and ungrammatical judgments, with a narrow range of variation within each category that is attributed to performance factors external to the system. By contrast, emergentist approaches assume probabilistically graded knowledge because grammatical knowledge is acquired from the input. Systematic IDs in both the cognitive systems supporting the acquisition of linguistic frequencies and patterns of individual experience with language will jointly predict variation in grammatical judgments, which will likely have a broader distribution than is predicted using the categorical approach.

A focus on IDs also has the potential to address issues concerning replicability [123]. Studies using the traditional experimental approach have tended to be underpowered. By contrast, IDs studies require many more participants, allowing a more-accurate and reliable estimate of the size of an effect in addition to how performance on the variable of interest varies in the sample (and, by inference, the population). At the same time, statistical modeling of ID data can be

\section{Box 2. Measurement of Individual Differences}

Research on IDs in language acquisition and processing poses significant methodological challenges. An experimental task that is reliable at the group level is, often by design, an unreliable ID task, because experimental tasks aim to minimize between-participant variability, whereas ID tasks should aim to maximize it [139]. Many common experimental psychology tasks exhibit low test/retest reliability for this reason. Low task reliability will attenuate the relationship between variables, which can cause both false negatives and false positives. First, if independent or dependent variables are measured unreliably, the correlation between independent and dependent variables will weaken and possibly disappear. Second, if covariates are measured unreliably, the relationship between independent and dependent variables can be artificially inflated [140]. Indeed, the use of experimental designs ill-suited for the study of IDs has been one proposed reason for the variable patterns of results on the relationship between bilingualism and executive functioning [141] as well as between language and statistical learning [133].

Measurement issues in IDs research extend beyond classical test theory notions, such as test/retest reliability. Most tasks in psycholinguistics are influenced by multiple cognitive processes that may combine in unintuitive ways. Isolating the process of interest for individual differences research will prove challenging without explicit process models for commonly used tasks. Take, for example, the lexical decision task, where participants typically decide whether a letter sequence (e.g., 'house or 'vouse') that appears on a computer is a word. Means and standard deviations of lexical decision tasks exhibit excellent test/retest reliability ( $r=0.87$ [142]). However, these raw means reflect the combination of many distinct cognitive parameters, such as encoding, evidence accumulation, response thresholds, and response execution times [143]. This means that two participants might have different means on a task despite being identical in the component process of interest, or have identical means despite differing in the component of interest. For example, while it is well known that children and older adults differ from young adults in lexical decision speed, parameter estimates from the drift diffusion model (a prominent cognitive process model of speeded decision making) show that older adults accumulate evidence at the same rate as college students but adopt more conservative response thresholds and take longer to execute a response [144], whereas children accumulate evidence more slowly in addition to adopting more-conservative response thresholds and taking longer to execute a response [145]. The development of similar process models for common psychological measures, such as the visual world paradigm [146], will be fruitful for careful individual differences research (see Outstanding Questions). 
challenging. The best ID studies test theoretical predictions and, thus, use constrained datafitting procedures to avoid fishing expeditions and p-hacking. This is not to say that exploratory analyses are not useful, but they must necessarily be followed up with subsequent, morefocused studies.

\section{Concluding Remarks}

We have argued that theoretical models of language acquisition and processing should be constrained by empirical demonstrations of IDs. Consistent with the existence imperative, we have argued that any theory must first and foremost predict meaningful IDs in language where they exist. As we have shown, IDs are pervasive across the entire linguistic system. A crucial future task will be to determine the range of IDs across different linguistic subsystems; for instance, how much variation is there in vocabulary compared with grammatical knowledge? While IDs in vocabulary are likely to be evident across the population and will be easily measureable, IDs in grammatical knowledge are likely to become restricted in range with growing experience, and may be most evident in adult speakers for uncommon structures or when measured using sensitive online methodologies (e.g., methodologies yielding reaction times, or brain-imaging techniques). Consistent with the environmental imperative, we have argued that theories of language acquisition and processing must account for the complex relationship between variation in input and language acquisition, which, as we have shown, leads to variation in language proficiency across the lifespan. The influence of linguistic experience on language is one of the key theoretical dimensions upon which theories of language acquisition and processing differ, owing to differing assumptions regarding innateness and the representational nature of linguistic operations. Thus, understanding how IDs in linguistic experience influence language can help test and refine theory. Finally, consistent with the architectural imperative, we have argued that IDs studies can reveal architectural properties of the linguistic system by identifying how particular components of language associate or dissociate with other components of the linguistic system and with other cognitive processes. The current literature suggests a good degree of interaction both within and outside of the language system.

While the study of IDs has been fruitful, there are considerable methodological challenges in marrying IDs and experimental designs (Box 2). However, innovations in data collection that allow increasingly large data sets (e.g., data sharing and online data collection platforms [124]) and the development of sophisticated statistical and computational methods have both made it easier to conduct IDs studies and increased the value of the information they yield. These developments point to an important reassessment of the implicit belief that language and other cognitive processes are subject to little meaningful variation.

\section{Acknowledgments}

Preparation of this manuscript was supported by funds from the Australian Research Council (CE40100041) to E.K. and from the Danish Council for Independent Research (FKK-grant DFF-7013-00074) to M.H.C. We thank Lindsey Drayton, Caroline Rowland, Julian Pine, and three anonymous reviewers for helpful comments, and Michael Frank for permission to use Figure $1 \mathrm{~A}$.

Resources

'http://wordbank.stanford.edu/

\section{References}

1. Farmer, T.A. et al. (2017) The inter-relationship between reading span task performance, linguistic experience, and the processing of unexpected syntactic events. Q. J. Exp. Psychol. (Hove) 70, 413-433

\section{Outstanding Questions}

How do potential internal sources of variation (e.g., WM, EF, or SL) interact? Studies of individual differences in language typically concentrate on one potential cognitive mechanism only (e. g., WM), but it is likely that all involve some overlapping skills (e.g., attention [2] or language skill [43]). Future studies will need to involve comprehensive batteries of tests. Imaging work will also be important in identifying common and separate neurological substrates.

What are the biological sources of IDs? Variation is a fundamental assumption of genetics; gene-environment interactions likely lead to IDs in neurological substrates that support language and associated cognitive processes [125]. Mapping from genes to brain to behavior is no small task [126], but investigating the problem from multiple perspectives will place important constraints on theoretical development.

How do internal sources of variation interact with the environment? How does variation in, for example, SL ability, interact with variation in quantity and quality of input? Do children with poor sequencing abilities require greater input to acquire components of language compared with their peers with better sequencing abilities (e.g., [127])?

How are IDs in acquisition related to IDs in adulthood? A focus on IDs across the lifespan is one way to better integrate the largely separate fields of acquisition and processing [128]. IDs in acquisition are stable across early development [31]. While there is likely to be a degree of continuity across the lifespan, other sources of variation likely contribute at different points of development (e.g., literacy instruction and reading frequency).

What kind of computational models can capture the full range of IDs? Although computational models are often damaged to simulate atypical language processing (e.g., [129]), there have been few attempts to model the full range of IDs we find in behavioral data (although see [19]). The importance of computational models to the cognitive sciences suggests that models of IDs should be made a priority. 
2. Kane, M.J. and Engle, R.W. (2002) The role of the prefrontal cortex in working-memory capacity, executive attention, and general fluid intelligence: an individual differences perspective. Psychon. Bull. Rev. 9, 637-671

3. Vogel, E. and Awh, E. (2008) How to exploit diversity for scientific gain: using individual differences to constrain cognitive theory. Curr. Dir. Psychol. Sci. 17, 171-176

4. Levinson, S.C. (2012) The original sin of cognitive science. Top. Cogn. Sci. 4, 396-403

5. Estes, W.K. (1956) Learning. Annu. Rev. Psychol. 7, 1-38

6. Cronbach, L.J. (1957) The two disciplines of scientific psychology. Am. Psychol. 12, 671-684

7. Sternberg, R.J. and Grigorenko, E.L. (2001) Unified psychology. Am. Psychol. 56, 1069-1079

8. Underwood, B.J. (1975) Individual differences as a crucible in theory construction. Am. Psychol. 30, 128-134

9. Bates, E. et al. (1988) From First Words to Grammar: Individual Differences and Dissociable Mechanisms, Cambridge University Press

10. Fodor, J. (1983) Modularity of Mind, The MIT Press

11. Hofmeister, P. et al. (2014) Processing effects in linguistic judgment data: (super-)additivity and reading span scores. Lang. Cogn. 6, 111-145

12. Sprouse, J. et al. (2012) A test of the relation between working memory and syntactic island effects. Language 88, 82-123

13. Lewis, R.L. et al. (2006) Computational principles of working memory in sentence comprehension. Trends Cogn. Sci. 10, $447-454$

14. van der Lely, H.K.J. and Pinker, S. (2014) The biological basis of language: insights from developmental grammatical impairment. Trends Cogn. Sci. 18, 586-595

15. Yang, C. et al. (2017) The growth of language: Universal Grammar, experience, and the principle of computation. Neurosci. Biobehav. Rev. 81, 103-119

16. Lidz, J. and Gagliardi, A. (2015) How nature meets nurture: universal grammar and statistical learning. Annu. Rev. Linguist. 1, 333-353

17. Lieven, E.V.M. (2016) Usage-based approaches to language development: where do we go from here? Lang. Cogn. 8, 346368

18. Bates, E. and MacWhinney, B. (1989) Functionalism and the competition model. In The Crosslinguistic Study of Sentence Processing (MacWhinney, B. and Bates, E., eds), pp. 3-76, Cambridge University Press

19. MacDonald, M.C. and Christiansen, M.H. (2002) Reassessing working memory: a comment on Just and Carpenter (1992) and Waters and Caplan (1996). Psychol. Rev. 109, 35-54

20. Christiansen, M.H. and Chater, N. (2016) Creating Language: Integrating Evolution, Acquisition, and Processing, MIT Press

21. Croft, W. (2017) Radical Construction Grammar: Syntactic Theory in Typological Perspective, Oxford University Press, (2001)

22. Goldberg, A. (2006) Constructions at Work: The Nature of Generalization in Language, Oxford University Press

23. Bates, E. and Goodman, J. (1997) On the inseparability of grammar and the lexicon: evidence from acquisition, aphasia and real-time processing. Lang. Cogn. Process. 12, 507-586

24. MacDonald, M.C. et al. (1994) The lexical nature of syntactic ambiguity resolution. Psychol. Rev. 101, 676-703

25. Kidd, E. et al. (2010) Lexical frequency and exemplar-based learning effects in language acquisition: evidence from sentential complements. Lang. Sci. 32, 132-142

26. Peter, M. et al. (2015) When and how do children develop knowledge of verb argument structure? Evidence from verb bias effects in a structural priming task. J. Mem. Lang. 81, 1-15

27. Chang, F. et al. (2006) Becoming syntactic. Psychol. Rev. 113, 234-272
28. Hart, B. and Risley, T. (1995) Meaningful differences in the everyday experience of young American children, Paul $\mathrm{H}$. Brookes

29. Weisleder, A. and Fernald, A. (2013) Talking to children matters: early language experience strengthens processing and builds vocabulary. Psychol. Sci. 24, 2143-2152

30. Bates, E. et al. (1995) Individual differences and their implications for theories of language development. In Handbook of child language (Fletcher, P. and MacWhinney, B., eds), pp. 96-151, Basil Blackwel

31. Bornstein, M. and Putnick, D.L. (2012) Stability of language in childhood: a multiage, multidomain, multimeasure, and multisource study. Dev. Psychol. 48, 477-491

32. Chonchaiya, W. et al. (2013) Developmental trends in auditory processing can provide early predictions of language acquisition in young infants. Dev. Sci. 16, 159-172

33. Brito, N.H. et al. (2016) Associations among family socioeconomic status, EEG power at birth, and cognitive skills during infancy. Dev. Cogn. Neurosci. 19, 144-15

34. Cristia, A. et al. (2013) Predicting individual variation in language from infant speech perception measures. Child Dev. 85, 13301345

35. Junge, C. et al. (2012) Rapid recognition at 10 months as a predictor of language development. Dev. Sci. 15, 463-473

36. Tsao, F.M. et al. (2004) Speech perception in infancy predicts language development in the second year of life: a longitudinal study. Child Dev. 75, 1067-1084

37. Frank, M.C. et al. (2017) Wordbank: an open repository fo developmental vocabulary data. J. Child Lang. 44, 677-694

38. Kidd, E. (2012) Individual differences in syntactic priming in language acquisition. Appl. Psycholinguist. 33, 393-418

39. O'Neill, D.K. (2007) The language use inventory for young children: a parent-report measure of pragmatic language development for 18- to 47-month-old children. J. Speech Lang. Hear. Res. 50, 214-228

40. Andrews, S. et al. (2017) Individual differences in automatic semantic priming J Exp. Psychol. Hum Percept. Perform. 43, 1025-1039

41. Kuperman, V. and Van Dyke, J.A. (2011) Effects of individual differences in verbal skills on eye-movement patterns during sentence reading. Cognition 65, 42-72

42. Andrews, S. (2015) Individual differences among skilled readers: the role of lexical quality. In The Oxford Handbook of Reading (Pollastek, A. and Treiman, R., eds), pp. 1-37, Oxford University Press

43. Wells, J. et al. (2009) Experience and sentence processing: statistical learning and relative clause comprehension. Cogn. Psychol. 58, 250-271

44. Swets, B. et al. (2007) The role of working memory in syntactic ambiguity resolution: a psychometric approach. J. Exp. Psychol. Gen. 136, 64-81

45. Just, M.A. and Carpenter, P.A. (1992) A capacity theory of comprehension: Individual differences in working memory. Psychol. Rev. 98, 122-149

46. Daneman, M.E. and Carpenter, P.A. (1980) Individual differences in working memory and reading. J. Verbal Learn. Verbal Behav. 19, 450-466

47. Mahowald, K. and Fedorenko, E. (2016) Reliable individual-leve neural markers of high level language processing: a necessary precursor for relating neural variability to behavioral and genetic variability. Neuroimage 139, 74-93

48. Pakulak, E. and Neville, H.J. (2011) Maturational constraints on the recruitment of early processes for syntactic processing J. Cogn. Neurosci. 23, 2752-2765

49. Dabrowska, E. (2015) Individual differences in grammatica knowledge. In Handbook of Cognitive Linguistics (Dabrowska, E. and Divjak, D., eds), pp. 650-668, De Gruyter 
50. Da?browska, E. (2008) The later development of an earlyemerging system: the curious case of the Polish genitive. Linguistics 46, 629-650

51. Street, J. and Da?browska, E. (2010) More individual differences in Language Attainment: how much do adult native speakers of English know about passives and quantifiers? Lingua 120, 2080-2094

52. Ambridge, B. et al. (2015) The ubiquity of frequency effects in first language acquisition. J. Child Lang. 42, 239-273

53. Reali, F. and Christiansen, M.H. (2007) Processing of relative clauses is made easier by frequency of occurrence. J. Mem. Lang. 57, 1-23

54. Arnon, I. et al. (2017) Digging up the building blocks of language: age-of-acquisition effects for multiword phrases. J. Mem. Lang. 92, 265-280

55. Hirsh-Pasek, K. et al. (2015) The contribution of early communication quality to low-income children's language success. Psychol. Sci. 26, 1071-1083

56. Rowe, M. (2012) A longitudinal exploration of the role of quantity and quality of child-directed speech in vocabulary development. Child Dev. 83, 1762-1774

57. Rowe, M. et al. (2016) Going beyond input quantity: Wh-questions matter for toddlers' language and cognitive development. Cogn. Sci. 41, 162-179

58. Jones, G. and Rowland, C.F. (2017) Diversity not quantity in caregiver speech: using computational modeling to isolate the effects of quantity and the diversity of the input on vocabulary growth. Cogn. Psychol. 98, 1-21

59. Hoff-Ginsberg, E. (1986) Function and structure in maternal speech: their relation to the child's development of syntax. Dev. Psychol. 22, 155-163

60. Hoff, E. (2003) The specificity of environmental influence: socioeconomic status affects early vocabulary development via maternal speech. Child Dev. 74, 1368-1378

61. Bradley, R.H. et al. (2001) The home environments of children in the United States part l: variations by age, ethnicity, and poverty status. Child Dev. 72, 1844-1867

62. Fernald, A. et al. (2012) SES differences in language processing skill and vocabulary are evident at 18 months. Dev. Sci. 16, 234248

63. Vasilyeva, M. et al. (2008) Emergence of syntax: commonalities and differences across children. Dev. Sci. 11, 84-97

64. Che, E. et al. (2017) Assessing the impact of conversational overlap in content on child language growth. J. Child Lang. 20, $1-25$

65. Troyer, M. and Borovsky, A. (2017) Maternal socioeconomic status influences the range of expectations during language comprehension in adulthood. Cogn. Sci. 41, 1405-1433

66. Rowe, M.L. and Goldin-Meadow, S. (2009) Differences in early gesture explain SES disparities in child vocabulary size at school entry. Science 323, 951-953

67. Eghbalzad, L. et al. (2016) Statistical learning ability can overcome the negative impact of low socioeconomic status on language development. In Proceedings of the 38th Annual Conference of the Cognitive Science Society (Papafragou, A., ed.), pp. 2129-2134, Cognitive Science Society

68. Nielsen, M. et al. (2017) The persistent samplingbias in developmental psychology: a call to action. J. Exp. Child Psychol. $162,31-38$

69. Schieffelin, B. and Ochs, E. (1986) Language socialization. Annu. Rev. Anthropol. 15, 163-191

70. Nielsen, M. and Haun, D. (2016) Why developmental psychology is incomplete without comparative and cross-cultural perspectives. Philos. Trans. R. Soc. Lond. B Biol. Sci. 371, 20150071

71. Darmin, N. and Drenowskli, A. (2008) Does social class predict diet quality? Am. J. Clin. Nutr. 87, 1017-1107

72. Drenowatz, C. et al. (2010) Influence of socio-economic status on habitual physical activity and sedentary behavior in 8- to 11 year-old children. BMC Public Health 10, 214
73. Brito, N.H. and Noble, K.G. (2014) Socioeconomic development and structural brain development. Front. Neurosci. 8, 276

74. Acheson, D.J. et al. (2008) New and updated tests of print exposure and reading abilities in college students. Behav. Res. Methods 40, 278-289

75. Christoffels, I. et al. (2006) Memory and language skills in simultaneous interpreters: the role of expertise and language proficiency. J. Mem. Lang. 54, 324-345

76. Henrich, J. et al. (2010) The weirdest people in the world? Behav. Brain Sci. 33, 61-135

77. Fernald, A. et al. (2006) Picking up speed in understanding: speech processing efficiency and vocabulary growth across the second year. Dev. Psychol. 42, 98-116

78. Dixon, J.A. and Marchman, V.A. (2007) Grammar and the lexcon: developmental ordering in language acquisition. Child Dev. $78,190-212$

79. Hoff, $\mathrm{E}$ et al. (2017) What explains the correlation between growth in vocabulary and grammar? New evidence from latent chance score analyses of simultaneous bilingual development. Dev. Sci. Published online February 22, 2017. http://dx.doi.org/ 10.1111/desc. 12536

80. Benard, M. et al. (2014) Individual differences in top-down restoration of interrupted speech: links to linguistic and cognitive abilities. J. Acoust. Soc. Am. 135, EL1-EL8

81. Carroll, R. et al. (2016) Age-related differences in lexical access relate to speech recognition in noise. Front. Psychol. 7,990

82. Trueswell, J.C. et al. (1993) Verb-specific constraints in sentence processing: separating effects of lexical preference from garden-paths. J. Exp. Psychol. Learn. Mem. Cogn. 19, 528-553

83. Conti-Ramsden, G. et al. (2001) Psycholinguistic markers of specific language impairment. J. Child Psychol. Psychiatry 42, 741-748

84. Gathercole, S.E. et al. (1992) Phonological memory and vocabulary development during the early school years: evidence from a longitudinal study. Dev. Psychol. 28, 887-898

85. Just, M.A. and Carpenter, P.A. (1992) A capacity theory of comprehension: individual differences in working memory. Psychol. Rev. 98, 122-149

86. Caplan, D. and Waters, G.S. (1999) Verbal working memory and sentence comprehension. Behav. Brain Sci. 22, 77-126

87. Ando, J. et al. (2001) Genetic structure of spatial and verbal working memory. Behav. Genet. 31, 615-624

88. van Leeuwen, M. et al. (2009) The genetic and environmenta structure of verbal and visuospatial memory in young adults and children. Neuropsychology 23, 792-802

89. Acheson, D. et al. (2011) A common neural substrate for language production and verbal working memory. J. Cogn. Neurosci. 23, 1358-1367

90. Jones, G. and Maken, B. (2015) Questioning short-term memory and its measurement: why digit span measures long-term associative memory. Cognition 144, 1-13

91. Van Dyke, J.A. et al. (2014) Low working memory capacity is only spuriously related to poor reading comprehension. Cognition 131, 373-403

92. McElree, B. et al. (2003) Memory structures that subserve sentence comprehension. J. Mem. Lang. 48, 67-91

93. Jones, G. et al. (2007) Linking working memory and long-term memory: a computational model of learning new words. Dev. Sci. 10, 853-873

94. Miyake, A. et al. (2000) The unity and diversity of executive functions and their contributions to complex 'frontal lobe' tasks: a latent variable analysis. Cogn. Psychol 4, 49-100

95. Friedman, N.P. et al. (2008) Individual differences in executive functions are almost entirely genetic in origin. J. Exp. Psychol. Gen. 137, 201-225 
96. Novick, J.M. et al. (2014) Clearing the garden-path: improving sentence processing through cognitive control training. Lang. Cogn. Neurosci. 29, 186-217

97. Vuong, L.C. and Martin, R.C. (2014) Domain-specific executive control and the revision of misinterpretations in sentence comprehension. Lang. Cogn. Neurosci. 29, 312-325

98. Woodard, K. et al. (2016) Taking your own path: individual differences in executive function and language processing skills in child learners. J. Exp. Child Psychol. 141, 187-209

99. Nozari, N. et al. (2016) The interplay of local attraction, context and domain-general cognitive control in activation and suppression of semantic distractors during sentence comprehension. Psychon. Bull. Rev. 23, 1942-1953

100. Trude, A. and Nozari, N. et al. (2017) Inhibitory control supports referential context use in language production and comprehension. In Proceedings of the 39th Annual Conference of the Cognitive Science Society (Gunzelmann, G., ed.), pp. 12181224, Cognitive Science Society

101. Khanna, M.M. and Boland, J.E. (2010) Children's use of language context in lexical ambiquity resolution. Q. J. Exp. Psychol. (Hove) 63, 160-193

102. Festman, J. et al. (2010) Individual differences in control of language interference in late bilinguals are mainly related to general executive abilities. Behav. Brain Funct. 6, 5

103. Engle, R.W. and Kane, M.J. (2004) Executive attention, working memory capacity, and a two-factor theory of cognitive control. Psychol. Learn. Motiv. 44, 145-199

104. McCabe, D.P. et al. (2010) The relationship between working memory capacity and executive functioning: evidence for a common executive attention construct. Neuropsychology 24, 222-243

105. Shafto, C.L. et al. (2012) Visual sequence learning in infancy: domain-general and domain-specific associations with language. Infancy 17, 247-271

106. Kidd, E. and Arciuli, J. (2016) Individual differences in statistical learning predict children's comprehension of syntax. Child Dev. 87, 184-193

107. Arciuli, J. and Simpson, I.C. (2012) Statistical learning is related to reading ability in children and adults. Cogn. Sci. 36, 286-304

108. Frost, R. et al. (2013) What predicts successful literacy acquisition in a second language. Psychol. Sci. 24, 1243-1252

109. Conway, C.M. et al. (2010) Implicit statistical learning in language processing: word predictability is the key. Cognition 114, 356-371

110. Daltrozzo, J. et al. (2017) Visual statistical learning is related to natural language ability in adults: an ERP study. Brain Lang. 166, $40-51$

111. Misyak, J.B. and Christiansen, M.H. (2012) Statistical learning and language: an individual differences study. Lang. Learn. 62, 302-331

112. Misyak, J.B. et al. (2010) Sequential expectations: the role of prediction-based learning in language. Top. Cogn. Sci. 2, 138153

113. Beckner, C. et al. (2009) Language is a complex adaptive system. Lang. Learn. 59 (Suppl. 1), 1-27

114. Gertner, Y. et al. (2006) Learning words and rules: abstract knowledge of word order in early sentence comprehension. Psychol. Sci. 17, 684-691

115. Yuan, S. et al. (2012) Counting the nouns: simple structural cues to verb meaning. Child Dev. 83, 1382-1399

116. Gleitman, L. (1990) The structural sources of verb meaning. Lang. Acquis. 1, 3-55

117. Abbot-Smith, K. and Tomasello, M. (2006) Exemplar-learning and schematization in a usage-based account of syntactic acquisition. Linguist. Rev. 23, 275-290

118. Gibson, E. and Fedorenko, E. (2013) The need for quantitative methods in syntax and semantics research. Lang. Cogn. Process. 28, 88-124
119. Lau, J.H. et al. (2017) Grammaticality, acceptability, and probability: a probabilistic view of linguistic knowledge. Cogn. Sci. 41, 1202-1241

120. Mahowald, K. et al. (2016) SNAP judgments: a small N acceptability paradigm (SNAP) for linguistic acceptability judgments. Language 93, 619-635

121. Phillips, C. (2010) Should we impeach armchair linguists? Jpn./ Korean Linguist. 17, 49-64

122. Sprouse, J. and Almeida, D. (2013) The empirical status of data in syntax: a reply to Gibson and Fedorenko. Lang. Cogn. Process. 28, 229-240

123. Open Science Collaboration (2015) Estimating the reproducibitity of psychological science. Science 349, 943-951

124. Stewart, N. et al. (2017) Crowdsouring samples in cognitive science. Trends Cogn. Sci. 21, 736-748

125. Mahowald, K. and Fedorenko, E. (2016) Reliable individual-leve neural markers of high level language processing: a necessary precursor for relating neural variability to behavioral and genetic variability. Neuroimage 139, 74-93

126. Dediu, D. and Christiansen, M.H. (2016) Language evolution: constraints and opportunities from modern genetics. Top. Cogn. Sci. 8, 361-370

127. Evans, J. et al. (2009) Statistical learning in children with specific language impairments. J. Speech Lang. Hear. Res. 52, 321-335

128. Christiansen, M.H. and Chater, N. (2017) Towards in integrated science of language. Nat. Hum. Behav. 1, 0163

129. Joannise, M.F. and Seidenberg, M.S. (1999) Impairments in verb morphology after brain injury: a connectionist model. Proc. Natl. Acad. Sci. U. S. A. 96, 7592-7597

130. Bishop, D. (2003) Test for the Reception of Grammar, Pearson Assessment

131. Saffran, J.R. et al. (1996) Statistical learning by 8-month-old infants. Science 274, 1926-1928

132. Milin, P. et al. (2017) A learning perspective on individual differences in skilled reading: exploring and exploiting orthographic and semantic discrimination cues. J. Exp. Psychol. Learn. Mem. Cogn. 43, 1730-1751

133. Siegelman, N. et al. (2017) Towards a theory of individual differ ences in statistical learning. Philos. Trans. R. Soc. Lond. B Biol. Sci. 372, 20160059

134. Siegelman, N. and Frost, R. (2015) Statistical learning as an individual ability: theoretical perspectives and empirical evidence. J. Mem. Lang. 81, 105-120

135. Onnis, L. and Thiessen, E. (2013) Language experience changes subsequent learning. Cognition 126, 268-284

136. Frost, R. et al. (2015) Domain generality vs. modality specificity: the paradox of statistical learning. Trends Cogn. Sci. 19, 117125

137. Isbilen, E. et al. (2017) Testing statistical learning implicitly: novel chunk-based measure of statistical learning. In Proceedings of the 39th Annual Conference of the Cognitive Science Society (Gunzelmann, G., ed.), pp. 2055-2060, Cognitive Science Society

138. Siegelman, N. et al. Re-defining 'learning' in statistical learning: what does an online measure reveal about the assimilation of visual regularities? Cogn. Sci. Published online October 7, 2017. https://doi.org/10.1111/cogs.12556

139. Hedge, C. et al. (2017) The reliability paradox: why robust cognitive tasks do not produce reliable individual differences. Behav. Res. Methods Published online July 19, 2017. http://dx. doi.org/10.3758/s13428-017-0935-1

140. Westfall, J. and Yarkoni, T. (2016) Statistically controlling for confounding constructs is harder than you think. PLoS One 11 e0152719

141. Costa, A. et al. (2014) On invisibility and experimental evidence. Biling. Lang. Cogn. 18, 25-26 
142. Yap, M.J. et al. (2012) Individual differences in visual word recognition: insights form the English Lexicon Project. J. Exp. Psychol. Hum. Percept. Perform. 38, 53-79

143. Ratcliff, R. et al. (2016) Diffusion decision model: current issues and history. Trends Cogn. Sci. 20, 260-281

144. Ratcliff, R. et al. (2010) Individual differences, aging, and IQ in two-choice tasks. Cogn. Psychol. 60, 127-157
145. Ratcliff, R. et al. (2012) Children are not like older adults: a diffusion model analysis of developmental changes in speeded responses. Child Dev. 83, 367-381

146. Altmann, G.T.M. and Kamide, Y. (1999) Incremental interpretation of verbs: restricting the domain of subsequent reference. Cognition 73, 247-269 